\title{
Estudio de la capacidad antioxidante y contenido de polifenoles en el proceso de clarificación del vino de flor de Jamaica (Hibiscus sabdariffa L.) utilizando cálices frescos.
}

\section{(Study of the antioxidant capacity and content of polyphenols in the clarification process of Jamaica flower (Hibiscus sabdariffa L.) wine)}

\author{
Zamora Cujilema Victoria ${ }^{1}$, Mariño Gabriel ${ }^{1}$, Carlos E. González Gallardoํㅜ, Jácome \\ Belén ${ }^{1}$, Beltrán-Sinchiguano Elena ${ }^{1}$
}

\begin{abstract}
Resumen:
El objetivo de esta investigación fue estudiar el efecto del proceso de clarificación por microfiltración tangencial (MFT) y por clarificación estática en frío a $6{ }^{\circ} \mathrm{C}$ por seis días, sobre el contenido de polifenoles y capacidad antioxidante del vino de flor de Jamaica (Hibiscus sabdariffa $L$ ) elaborado a partir de cálices frescos. Se realizaron análisis fisicoquímicos (sólidos solubles, $\mathrm{pH}$, turbidez, acidez titulable expresada como ácido tartárico y málico, color y turbidez) y bioquímicos por espectrofotometría del contenido de polifenoles totales (método Folin-Ciocalteu) y de la capacidad antioxidante (método TEAC). La capacidad antioxidante del mosto fermentado sin tratamiento de clarificación fue superior al mosto fermentado clarificado por MFT a 2 bar e inferior al mosto fermentado clarificado en frío a $6^{\circ} \mathrm{C}$ y por seis días. La actividad antioxidante del mosto fermentado clarificado por MFT a 2 bar es inferior en comparación con el proceso de clarificación estática en frío con una actividad antioxidante de $8.21 \mathrm{mM}$ equivalentes de Trolox/ $100 \mathrm{ml}$. El proceso de clarificación en frío a $6^{\circ} \mathrm{C}$ y por seis días contiene mayor capacidad antioxidante, pero en función a la limpidez (NTU) como atributo de calidad es baja en comparación al vino obtenido por microfiltración tangencial a 2 bar.
\end{abstract}

Palabras clave: vino; vino de flor de Jamaica; compuestos fenólicos; antioxidantes; tecnología de membranas; microfiltración tangencial; bebidas alcohólicas.

\begin{abstract}
:
The objective of this research was to study the effect of the clarification process by tangential microfiltration (MFT) and the static clarification in cold at $6{ }^{\circ} \mathrm{C}$ for six days, on the content of polyphenols and the antioxidant capacity of the Jamaica flower (Hibiscus sabdariffa $\mathrm{L}$ ) wine, made from fresh chalices. Physicochemical analyzes (soluble solids, $\mathrm{pH}$, turbidity, titratable acidity expressed as tartaric and malic acid, color and turbidity) and biochemical analyzes were performed by spectrophotometry of total polyphenols content (Folin-Ciocalteu method) and antioxidant capacity (TEAC method). The antioxidant capacity of the fermented wort without clarification treatment was superior to the fermented wort clarified by MFT at 2 bar and inferior to the fermented wort clarified in cold at $6{ }^{\circ} \mathrm{C}$ and for six days. The antioxidant activity of the fermented must clarified by MFT at 2 bar is lower compared to the cold static clarification process with an antioxidant activity of $8.21 \mathrm{mM}$ of Trolox equivalent / $100 \mathrm{ml}$. The process of clarification in cold at $6{ }^{\circ} \mathrm{C}$ and for six days contains greater capacity of antioxidant, but in function of the cleaning (NTU) as attribute of quality is low in comparison to the one obtained by tangential microfiltration to 2 bar.
\end{abstract}

Keywords: wine; Jamaica flower wine; polyphenol content; antioxidant activity; cold static clarification; tangential microfiltration.

\footnotetext{
${ }^{1}$ Universidad Tecnológica Equinoccial, Facultad de Ciencias de la Ingeniería, Quito Ecuador, (elena.beltran@ute.edu.ec)
} 


\section{Introducción}

La flor de Jamaica (Hibiscus sabdariffa L) también llamada rosa de Jamaica o Rosella es una planta perteneciente a la familia de las Malváceas, cultivada en zonas con climas tropicales y subtropicales (Sáyago \& Goñi, 2010) de América Central especialmente en México en el estado de Guerrero, América del Sur, India, Malasia y en el sureste asiático hasta el sur de China (Medina \& Sumaya, 2013); en el Ecuador la producción de flor de Jamaica se encuentra limitada únicamente a la región Amazónica. Son cultivadas con el propósito de usar el cáliz de sus flores para producir té y refrescos altamente apreciados por su particularidad de trasmitir frescura y por su alto contenido en compuestos bioactivos como antocianinas en un $1.5 \%$, ácidos orgánicos en un 15-30\%, polisacáridos mucilaginosos en un $50 \%$, así como flavonoides y fotoesteroles (Castañeda \& Cáceres, 2014).

Con el fin de innovar y aprovechar las propiedades funcionales que ofrecen los cálices frescos de flor de Jamaica, se utilizó este cultivo para la producción de vino con el objetivo de estudiar el efecto del proceso de clarificación por microfiltración tangencial a 2 bar y por clarificación estática en frío a $6{ }^{\circ} \mathrm{C}$ por seis días en el contenido de polifenoles y capacidad antioxidante del vino de los cálices frescos de flor de Jamaica. Se determinó el proceso de clarificación (microfiltración tangencial y clarificación estática en frío) del vino de flor de Jamaica obtenido a partir de cálices frescos que contengan la mayor capacidad antioxidante a través del método Folin-Ciocalteu's y contenido de polifenoles mediante el método TEAC y se evaluaron las características físico-químicas del vino de flor de Jamaica con la mayor capacidad de antioxidantes y contenido de polifenoles.

\section{Metodología}

\subsection{Proceso de microfiltración tangencial}

Se realizó después del proceso de trasiego a 2 bar y a una temperatura de $21^{\circ} \mathrm{C}$, empleando el sistema de microfiltración tangencial con un primer módulo de refrigeración y un segundo módulo que contiene a la membrana de MFT de cerámica ( $\alpha$ - alúmina), con un tamaño de poro de $0.45 \mu \mathrm{m}, 19$ canales de diámetro de $6 \mathrm{~mm}$ con $1.02 \mathrm{~m}$ de longitud y una superficie de filtrado de $0.36 \mathrm{~m}^{2}$. Se procedió a determinar la permeabilidad de la membrana para lo cual se utilizó agua destilada y se trabajó bajo presiones de $1.5 ; 2 ; 2.5$; y 3 bar, con el fin de tomar en cuenta factores como el flujo de permeado, volumen de alimentación, masa de retenido y permeado, tiempo de operación, presiones de entrada y salida para calcular parámetros de flujo transmembrana (JP), presión transmembrana (PTM) y factor de retención volumétrica (FRV).

\subsection{Proceso de clarificación estática en frío}

El vino trasegado fue colocado en refrigeración durante 6 días en absoluto reposo a $6{ }^{\circ} \mathrm{C}$. Durante este proceso y para cada día de clarificación se tomaron muestras de la parte superior del vino para efectuar análisis correspondientes de color, turbidez, contenido de polifenoles y capacidad antioxidante.

\subsection{Determinación del contenido de polifenoles totales}

El contenido de polifenoles se basó en el uso del reactivo de Folin-Ciocalteu descrito por Georgé, Brat, Alter \& Amiot (2005). Este análisis se efectuó para los cálices frescos de flor de Jamaica, mosto inicial, mosto fermentado, vino microfiltrado, retenido, vino clarificado por frío y para las muestras tomadas durante el proceso de MFT y de clarificación en frío. La capacidad antioxidante se determinó por espectrofotometría, mediante el método TEAC que está basado en la decoloración del radical ABTS según la 
metodología desarrollada por, Pellegrini, Proteggiente, Pannala, Yang \& Rice (1999), adaptado a las condiciones de la Universidad Tecnológica Equinoccial.

\subsection{Análisis físico - químicos}

Se realizó el análisis físico-químico del vino de cálices de flor de Jamaica de acuerdo con la norma INEN NTE 374 para vinos de frutas. Los análisis y la metodología aplicada se pueden observar en la Tabla 1.

Tabla 1. Metodología para la caracterización físico-química del vino de los cálices frescos de la flor de Jamaica

\begin{tabular}{lcc}
\hline \multicolumn{1}{c}{ Parámetros } & Unidades & Método de Referencia \\
\hline Grado alcohólico & ${ }^{\circ} \mathrm{GL}$ & INEN 340 \\
Metanol & $\mathrm{mg} / \mathrm{l}$ & INEN 347 \\
Anhídrido sulfuroso libre & $\mathrm{g} / \mathrm{l}$ & INEN 357 \\
Anhídrido sulfuroso total & $\mathrm{g} / \mathrm{l}$ & INEN 356 \\
Sólidos Solubles & ${ }^{\circ} \mathrm{Bx}$ & $\mathrm{AOAC} 932.12$ \\
& Ácido Málico & \\
Acidez Titulable & Acido Tartárico & AOAC 942.15 \\
$\mathrm{pH}$ & & AOAC 945.27 \\
\hline
\end{tabular}

\subsection{Medición de color y turbidez}

Para la medición de color se tomaron $2 \mathrm{ml}$ de las muestras y se las colocó en cubetas de $4 \mathrm{ml}$ para espectrofotómetro, luego se procedió a realizar un barrido manual en un rango espectral de absorbancias de 380 a $780 \mathrm{~nm}$ a intervalos de $10 \mathrm{~nm}$. Las mediciones obtenidas fueron analizadas mediante el modelo matemático de Glories descrito en la OIVMABS-26 para determinación de la intensidad del color en vinos (OIV, 2009). Para determinar la turbidez de las muestras de microfiltración y de clarificación estática en frío se empleó el método internacional OIV-MA-AS2-08, para determinación de turbidez en vinos mediante el análisis Nefelométrico (OIV, 2009).

\subsection{Diseño experimental}

El análisis estadístico se realizó con el programa InfoStat versión 2016 con un diseño completamente al azar, para determinar la comparación del proceso de clarificación por microfiltración tangencial efectuada a 2 bar y de la clarificación estática en frío después de 6 días de reposo sobre contenido de polifenoles, capacidad antioxidante, color y turbidez. El análisis de varianza se realizó con ANOVA simple, mediante la prueba de Tukey, con un nivel de confianza del $95 \%$.

\section{Resultados y discusión}

\subsection{Proceso de microfiltración tangencial del vino}

En la Tabla 2 se muestran las características físico-químicas del mosto fermentado antes (alimentación), durante (microfiltración) y al finalizar (retenido) el proceso de microfiltración tangencial.

Es evidente que la concentración de sólidos solubles y ácido málico presentes en la alimentación disminuyen en el permeado, pero se concentran en el retenido, indicando que estos compuestos lograron traspasar los poros de la membrana libremente por tener un bajo peso molecular según lo establecido por Bhave (2014), y lo reportado por Cassano, Conidi \& Drioli (2010) en su estudio de la clarificación de jugo de cactus (Opuntia ficusindica) al obtener un comportamiento similar. 
Tabla 2.Características físico-químicas del proceso de MFT

\begin{tabular}{ccccc}
\hline \multirow{2}{*}{ Parámetros } & \multirow{2}{*}{ Unidad } & \multicolumn{3}{c}{ Resultados } \\
\cline { 2 - 5 } & & Alimentación & Permeado & Retenido \\
\hline Grado Alcohólico & ${ }^{\circ} \mathrm{Gl}$ & 6 & 6 & 6 \\
Sólidos Solubles & ${ }^{\circ} \mathrm{Bx}$ & $15.15 \pm 0.07$ & $14.30 \pm 0.20$ & $15.20 \pm 0.40$ \\
$\mathrm{pH}$ & & $2.38 \pm 0.00$ & $2.38 \pm 0.02$ & $2.40 \pm 0.05$ \\
Acidez Titulable & $\mathrm{g} / \mathrm{l}$ ácido málico & $8.14 \pm 0.05$ & $7.30 \pm 0.03$ & $7.81 \pm 0.11$ \\
Turbidez & $\mathrm{NTU}$ & $748.00 \pm 0.71$ & $1.33 \pm 0.02$ & $>1000$ \\
\hline
\end{tabular}

El proceso de microfiltración del vino de los cálices de flor de Jamaica a 2 bares de presión inició con 15 litros de alimentación, duró 37.43 minutos antes que la membrana se colmatara por completo. Los parámetros o factores obtenidos que fundamentan a la microfiltración tangencial del vino de flor de Jamaica se muestran en la Tabla 3.

Tabla 3.Parámetros de la MFT obtenidos en el Vino

\begin{tabular}{lll}
\hline \multicolumn{1}{c}{ Parámetros o Factores } & Unidades & Resultado \\
\hline Presión de Entrada $\left(P_{e}\right)$ & bar & 2.06 \\
Presión de Salida $\left(P_{s}\right)$ & bar & 2.13 \\
Presión de Permeado $\left(P_{p}\right)$ & bar & 0 \\
Presión transmembrana $(\mathrm{PMT})$ & bar & 2.09 \\
Volumen de Alimentación $\left(V_{a}\right)$ & $l$ & 15 \\
Volumen de Retenido $\left(V_{r}\right)$ & $l$ & 5 \\
Volumen de Permeado $\left(V_{p}\right)$ & $l$ & 10 \\
Rendimiento & $\%$ & 66.67 \\
Factor de Retención Volumétrica $(\mathrm{FRV})$ & & 3 \\
Velocidad Tangencial $(U)$ & $l . h^{-1} \cdot m^{-2}$ & 13.89 \\
\hline
\end{tabular}

El curso del flujo de permeado en función al tiempo y al Factor de Retención Volumétrica (FRV) a una concentración de 2 bar y $20^{\circ} \mathrm{C}$ (Figura 1) reflejan que el flujo de permeado inicial fue de $71.13 \mathrm{l} / \mathrm{hm}^{2}$ disminuyendo gradualmente con el tiempo, debido a la formación de gel según lo establecido por El Rayess, Albasi, Bacchin, Taillandier \& Mietton (2011) que menciona que en la microfiltración de vino la concentración de moléculas en la superficie aumenta y las moléculas alcanzan su límite de solubilidad y precipitan sobre la membrana para formar un gel sólido pero incrementando el FRV.

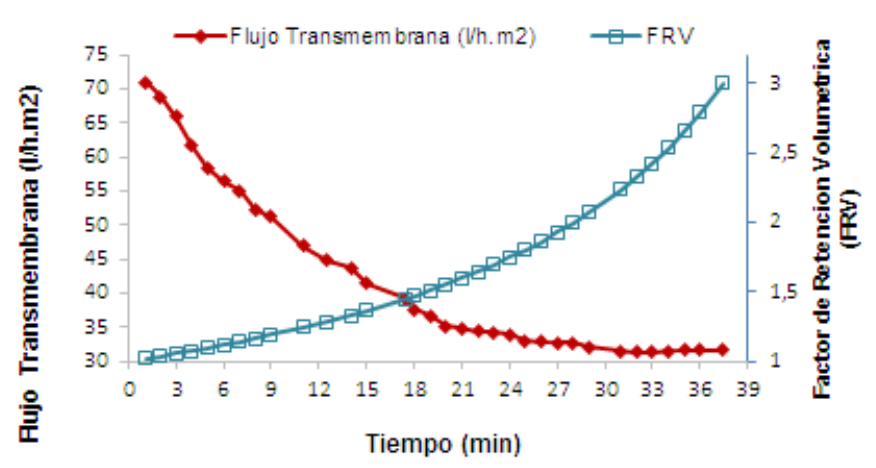

Figura 1.Flujo transmembrana y FRV en función del tiempo

Una reducción del $77.71 \%$ del flujo inicial fue obtenida después de 37.43 min., con un FRV de 3, en comparación con lo obtenido por Ennouri et al., (2015) en su estudio sobre la clarificación de jugo de zanahoria morada donde la reducción fue del 95 \% a 350 min con factor de retención de 1.8 a una presión de 1.8 bar $(180 \mathrm{kPa})$. Cisse, Vaillant, SoroDoudjo, Reynes \& Dornier (2011) establecen que a altos factores de retención volumétrica $(>1)$ el flujo de permeado permanece proporcional a la presión transmembrana, lo cual permitiría predecir el flujo de permeado en función de la presión transmembrana y la relación de reducción volumétrica y mencionan que si el FRV es constante y creciente, 
los parámetros de MFT son aceptados para aplicarlos a fines industriales, en nuestro caso la presión de 2 bar supera a los $50 \mathrm{l} / \mathrm{hm}^{2}$ (Flujo transmembrana) a 2.1 bar, aceptable para condiciones industriales por ser proporcional al flujo transmembrana.

\subsection{Proceso de clarificación estática en frío}

En la Tabla 4 se presentan los resultados obtenidos del proceso de clarificación estática en frío a $6{ }^{\circ} \mathrm{C}$ durante 6 días. Según Jackson, Kilcast \& Subramaniam (2011) al emplear temperaturas bajas se logra prolongar la vida útil del vino y retrasar la mayoría de las reacciones químicas como la precipitación del ácido tartárico a cristales, impidiendo que disminuya su contenido en el vino; se refleja que el ácido tartárico tiene una ligera variación en comparación con el vino trasegado, pero no existió diferencia significativa ( $p$ $>0.05$ ) durante su análisis por lo tanto la concentración de este ácido se mantiene constante.

La concentración de sólidos solubles $\left({ }^{\circ} \mathrm{Bx}\right)$ fue muy similar en el vino trasegado y en la clarificación estática en frío, no hubo diferencias significativas entre ellos $(p>0.05)$, lo cual implica que la clarificación estática en frío no modificó el contenido inicial de Sólidos Solubles $\left({ }^{\circ} \mathrm{Bx}\right)$.

En el proceso de clarificación estática en frío el contenido de grado alcohólico se mantuvo constante, mientras que en el pH se mantuvo ligeramente estable de 2.38 a 2.41. Jackson, Kilcast \& Subramaniam (2011) mencionan que en períodos de almacenamiento corto existen ligeras variaciones de $\mathrm{pH}$ debido a que los componentes que forman parte del vino se estabilizan a nuevas condiciones de reacción y que a pH inferiores a 3 permiten la estabilidad o crecimiento nulo de bacterias ácido lácticas.

Tabla 4. Datos obtenidos del proceso por clarificación estática en frío

\begin{tabular}{cccc}
\hline \multirow{2}{*}{ Parámetros } & Unidad & \multicolumn{2}{c}{ Resultados } \\
\cline { 3 - 4 } & & $\begin{array}{c}\text { Mosto } \\
\text { Fermentado }\end{array}$ & $\begin{array}{c}\text { Clarificación estática } \\
\left.\text { en frío (6 }{ }^{\circ} \mathbf{C}\right)\end{array}$ \\
\hline Grado Alcohólico & ${ }^{\circ} \mathrm{GL}$ & 6 & 6 \\
Sólidos Solubles & ${ }^{\circ} \mathrm{Bx}$ & $15.15 \pm 0.07$ & $15.10 \pm 0.01$ \\
$\mathrm{pH}$ & & $2.38 \pm 0.00$ & $2.41 \pm 0.01$ \\
Acidez Titulable & $\mathrm{g} / \mathrm{L}$ ácido málico & $8.14 \pm 0.05$ & $7.97 \pm 0.01$ \\
Acidez Titulable & $\mathrm{g} / \mathrm{L}$ ácido tartárico & $9.11 \pm 0.05$ & $8.93 \pm 0.18$ \\
Turbidez & $\mathrm{NTU}$ & $748.00 \pm 0.71$ & $61.70 \pm 0.07$ \\
\hline
\end{tabular}

\subsection{Determinación del contenido de polifenoles totales}

Los resultados sobre el contenido de polifenoles totales en el proceso de vinificación de los cálices frescos de flor de Jamaica indican que existe diferencia significativa entre los cálices, el mosto sin fermentar y el mosto fermentado (Figura 2).

Ifie, Marshall, Ho \& Williamson (2016) en su estudio sobre la vinificación de flor de Jamaica durante 40 días de fermentación, reportan $226.0 \mathrm{mg}$ equivalente de ácido gálico/100 ml de muestra en los cálices, $74.3 \mathrm{mg}$ equivalente de ácido gálico/100 ml de muestra en el mosto sin fermentar y $136.3 \mathrm{mg}$ equivalente de ácido gálico/100 ml de muestra en el mosto fermentado; los resultados obtenidos indican que a pesar del bajo contenido de polifenoles en los cálices de flor de Jamaica el mosto fermentado presenta una concentración de polifenoles próxima al vino obtenido por Ifie, Marshall, Ho \& Williamson (2016). 


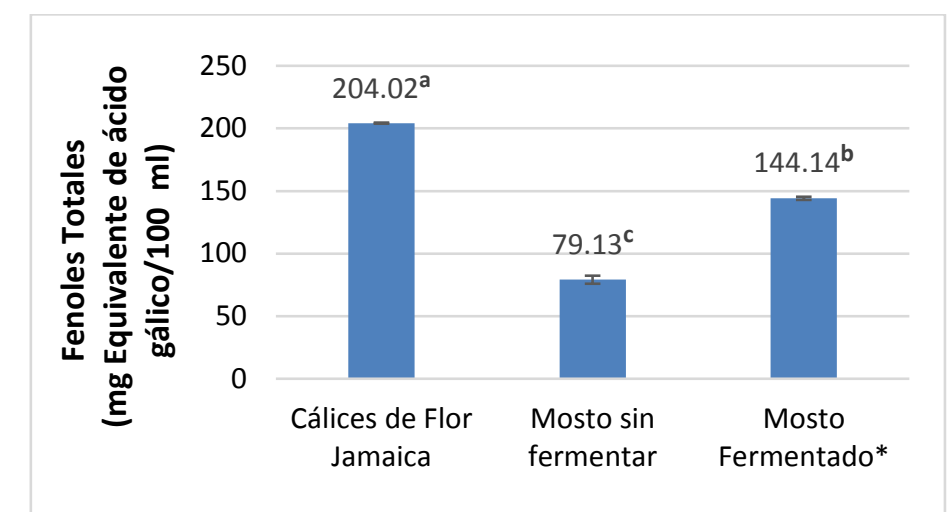

Figura 2.Contenido de Polifenoles en el proceso de vinificación * Representa la muestra de alimentación en el MFT, de Clarificación estático en frío día 0

Las medias con letras distintas indican diferencia significativa de acuerdo con la prueba de Tukey $(\mathrm{p}<0.05) ; \mathrm{n}= \pm 4$ Desviación estándar

La concentración de polifenoles totales del vino de flor de Jamaica es menor basada en los valores obtenidos para vinos orgánicos (Cabernet-Sauvignon, Rosso Veronese, Merlot, Cabernet Franc) de $441.7 \mathrm{mg}$ Eq. Acid. Gálico/100ml de muestra y vinos convencionales (Barbera, Refosco, Valpolicella, Teroldego) de $422.57 \mathrm{mg}$ Eq. Acid. Gálico/100ml de muestra según lo reportado por Garaguso \& Nardini (2015) en su estudio de la capacidad antioxidante y contenido de polifenoles en vinos orgánicos y convencionales donde determina que la alta concentración de polifenoles en un vino se debe a la calidad de la materia prima y al proceso de fermentación ejecutado. El Rayess, Albasi, Bacchin, Taillandier, \& Mietton (2011) mencionan que durante la fermentación de vinos tintos, los compuestos fenólicos pueden reaccionar entre sí (tanino-antocianina); se dan interacciones fisicoquímicas entre compuestos fenólicos (taninos-tanino y antocianinaantocianina) o reacciones con otros compuestos (proteínas de tanino) y reacciones de taninos con ciertos flavonoides que conforman la gran mayoría de los compuestos fenólicos en el vino por lo cual la contracción de polifenoles en un vino aumenta, lo cual conlleva a determinar que en el vino de los cálices de flor de Jamaica las reacciones entre los compuestos fenólicos son incompletas en comparación con vinos elaborados a partir de uva.

El proceso de clarificación estática en frío a $6{ }^{\circ} \mathrm{C}$ presentó el mayor contenido de polifenoles (139.32 mg equivalente de ácido gálico por $100 \mathrm{ml}$ de muestra); se encontraron diferencias significativas $(p<0.05)$ con el proceso de clarificación por MFT a 2 bar $(116.88$ mg Equivalente de ácido gálico por $100 \mathrm{ml}$ de muestra) y con el mosto fermentado (144.14 mg Equivalente de ácido gálico por $100 \mathrm{ml}$ de muestra) (Figura 3).Arriagada, Saenz \& Bordeu (2005) mencionan que durante la microfiltración existe una disminución del $10 \%$ de compuesto fenólicos en comparación con el $5 \%$ que se desarrolla en la clarificación en frío reportado por Cisse, Vaillant, SoroDoudjo, Reynes \& Dornier (2011).

En la Figura 4 se observa que el comportamiento de los compuestos fenólicos durante el proceso de microfiltración tangencial es variable, según El Rayess, Albasi, Bacchin, Taillandier \& Mietton (2011), en condiciones dinámicas de un proceso de MFT es normal la variación de compuestos fenólicos debido a que tienden a acumularse en la entrada de los poros en el lado de alimentación de la membrana ya sea por la cantidad o por la naturaleza de las moléculas, lo cual provoca un incremento de la energía cinética en la superficie de la membrana, energía que tiene una fuerte incidencia en el ensuciamiento de la membrana durante la microfiltración del vino lo cual impide la salida de polifenoles hacia el permeado. 


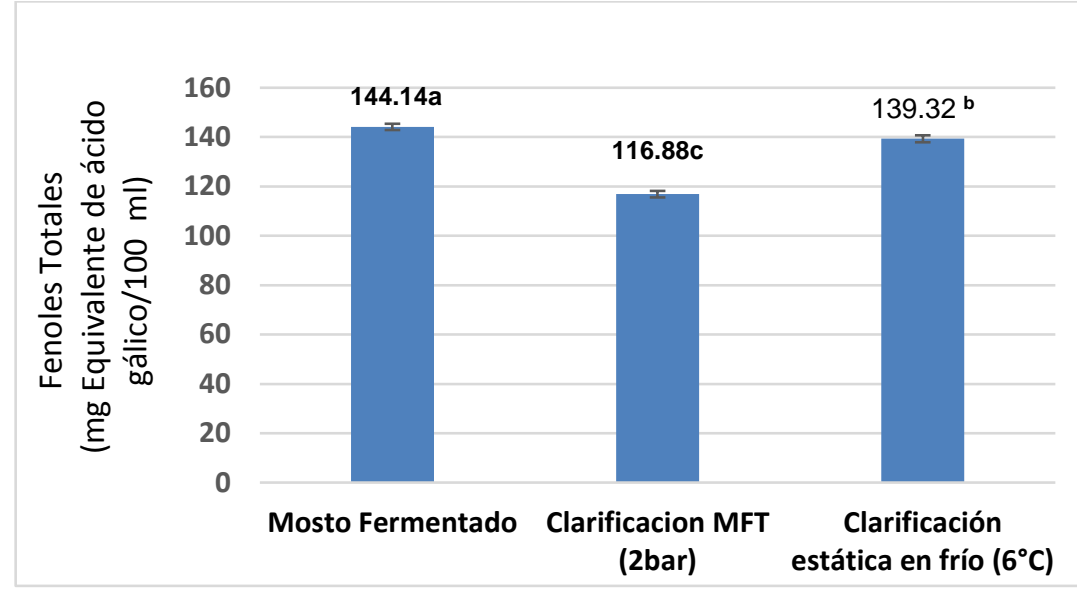

Figura 3. Contenido de polifenoles en el proceso de clarificación por MTF y por clarificación estática en frío $\left({ }^{\circ} \mathrm{C}\right)$

Las medias con letras distintas indican diferencia significativa de acuerdo con la prueba de Tukey $(\mathrm{p}<$

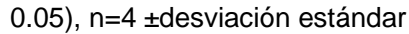

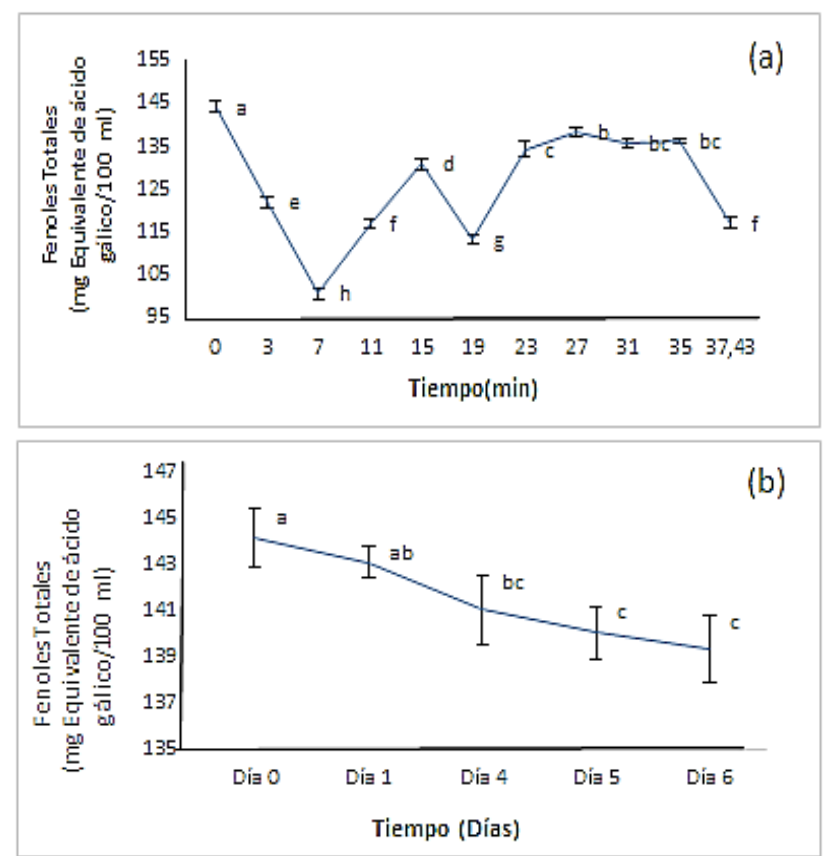

Figura 4. Comportamiento de los compuestos fenólicos: (a) Proceso de MFT (2bar), (b) Clarificación estática en frío

Las medias con letras distintas indican diferencia significativa de acuerdo con la prueba de Tukey $(\mathrm{p}<0.05), \mathrm{n}=4$ \pm desviación estándar

Los resultados estadísticos obtenidos durante los días de clarificación en frío (Figura 7) determinan que existen diferencias significativas entre la concentración de polifenoles del día 0 con $144.14 \mathrm{mg}$ Eq. Acid. Gálico/ para el día 1 en $141.53 \mathrm{mg}$ Eq. Acid. Gálico/ml disminuyendo en un $2 \%$ a las $24 \mathrm{~h}$, para el día 4 en $140.08 \mathrm{mg} \mathrm{Eq.} \mathrm{Acid.} \mathrm{Gálico/100} \mathrm{ml,}$ para el día 5 en $139.35 \mathrm{mg}$ Eq. Acid. Gálico/ml, y para el día 6 en $139.32 \mathrm{mg} \mathrm{Eq}$. Acid. Gálico/ml. Bimpilas, Tsimogiannis, Balta-Brouma, Lymperopoulou \& Oreopoulou (2015) establecen que durante la clarificación en frío la concentración de compuestos polifenólicos disminuye considerablemente después de las $24 \mathrm{~h}$ y paulatinamente conforme transcurre al producirse reacciones de polimerización (antocianinas-procianidinas) y no de oxidación entre las sustancias fenólicas. 


\subsection{Determinación de la capacidad antioxidante MÉTODO TEAC}

Los resultados de los análisis de capacidad antioxidante en el proceso de vinificación de lo cálices de flor de Jamaica (Figura 5), señalan que existe diferencia significativa entre los cálices (13.74 mM Trolox/ 100ml de muestra), mosto sin fermentar (4.56 mM Trolox/ $100 \mathrm{ml}$ de muestra) y mosto fermentado (9.67 mM Trolox/ 100ml de muestra).

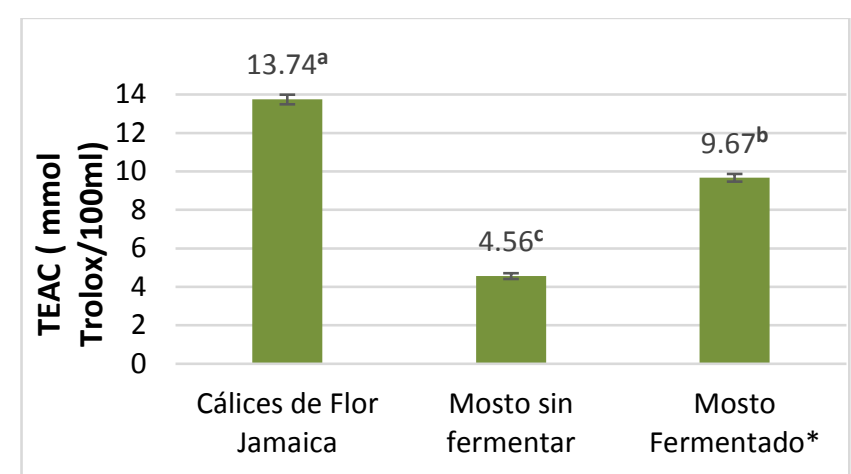

Figura 5.Capacidad Antioxidante en el proceso de vinificación y clarificación por MFT (2bar) y clarificación estática en frío

* Representa la muestra de alimentación en el MFT, de Clarificación estático en frío día 0 Las medias con letras distintas indican diferencia significativa de acuerdo con la prueba de Tukey $(p<0.05) ; n= \pm 4$ Desviación estándar

La capacidad antioxidante detectada de los cálices de 13,740 $\mu \mathrm{M}$ equivalentes de Trolox/ $100 \mathrm{~g}$ por extracción en acetona, es superior a los resultados reportados por Borrás, Fernández, Arráez, Palmeros, Del Val-Díaz, Andrade, Fernández, Gómez \& Segura (2015) en su estudio sobre la caracterización de compuestos fenólicos en diferentes variedades de Hibiscus sabdariffa en extractos etanólicos (Tabla 5) e inferior a 16,000 $\mu \mathrm{M}$ equivalentes de Trolox/100 g detectada por extracción acuosa según lo reportado por Fernández, Rodríguez, Beltrán, Pasini \& Fernández (2011).

El vino de flor de Jamaica (mosto fermentado) presenta una actividad antioxidante superior de $9.67 \mathrm{mM}$ equivalentes de Trolox/100 ml en comparación con los valores obtenidos para vinos orgánicos (Cabernet-Sauvignon, Rosso Veronese, Merlot, Cabernet Franc) de $2.37 \mathrm{mM}$ equivalentes de Trolox/100 ml y para vinos convencionales (Barbera, Refosco, Valpolicella, Teroldego) de $1.88 \mathrm{mM}$ equivalentes de Trolox/ $100 \mathrm{ml}$ de muestra según lo reportado por Garaguso \& Nardini (2015) al indicar que tanto en grupos orgánicos como convencionales los polifenoles totales son los principales responsables de la actividad antioxidante de los vinos.

De acuerdo con el análisis estadístico de la actividad antioxidante de ambos procesos de clarificación, se indica que existen diferencias significativas entre los procesos de clarificación por MFT a 2 bar y por clarificación estática en frío a $6^{\circ} \mathrm{C}$ por seis días al cual fue expuesto el mosto fermentado (Figura 6) y se determinó que la mayor actividad antioxidante se encuentra en el vino obtenido por clarificación estática en frío a $6{ }^{\circ} \mathrm{C}$ con una actividad de $8.21 \mathrm{mM}$ Trolox $/ 100 \mathrm{ml}$ en comparación a la concentración de $6.4 \mathrm{mM}$ Trolox/100 ml del proceso de clarificación de MFT a 2 bar con respecto a la concentración de $9.67 \mathrm{nM}$ presente en el mosto fermentado.

Los valores obtenidos durante el proceso de microfiltración de $6.40 \mathrm{mM}$ Trolox/100 $\mathrm{ml}$ a 2 bar son menores a lo reportado por Gonzáles (2013) de $42.62 \mathrm{mM}$ Trolox/100 ml a 2 bar en el vino de mora microfiltrado; el valor obtenido es inferior debido a que el mosto fermentado presenta una mayor concentración de coloides (748.00 NTU) que colmatan rápidamente la membrana del equipo durante el proceso, según El Rayess, Albasi, Bacchin, Taillandier \& Mietton (2011) el vino tinto presenta entre 1.5 a $6 \mathrm{~g} / \mathrm{l}$ de coloides (compuestos fenólicos). El vino retenido presentó una mayor actividad antioxidante de $10.21 \mathrm{mM}$ Trolox/100 ml, superior al vino microfiltrado y al vino por clarificación estática en 
frío que reflejó un valor de $8.21 \mathrm{mM}$ Trolox/100 ml, Jackson, Kilcast, \& Subramaniam (2011) establecen que las temperaturas bajas ayudan a la precipitación rápida de los coloides en suspensión después de las 24 h, pero se mantienen constantes a temperaturas estables.

Tabla 5. Contenido de compuestos polifenólicos totales y actividad antioxidante en extractos de Hibiscus sabdariffa

\begin{tabular}{llcc}
\hline Variedad & Origen & $\begin{array}{c}\text { Contenido de } \\
\text { Polifenoles totales } \\
(\mathbf{m g} \text { Ácido } \\
\text { Gálico/100 } \mathbf{~ m l ~ o ~ g ) ~}\end{array}$ & $\begin{array}{c}\text { Actividad Antioxidante } \\
(\boldsymbol{\mu m o l} \text { Equivalente de } \\
\text { Trolox /100 } \mathbf{~ m l ~ o ~ g ) ~}\end{array}$ \\
\hline Americana & Jalisco & $30 \pm 6$ & $104.0 \pm 9$ \\
Tepalcatepec & Michoacán & $90 \pm 20$ & $83.0 \pm 0.6$ \\
Diamante & Jalisco & $98 \pm 1$ & $112.0 \pm 8$ \\
Tempranilla & Colima & $45 \pm 9$ & $84.0 \pm 2$ \\
P. Anzar & Colima & $28 \pm 4$ & $97.3 \pm 8$ \\
Real & Veracruz & $100 \pm 4$ & $101.0 \pm 6$ \\
Reyna & Nayarit & $70 \pm 1$ & $97.0 \pm 4$ \\
Quesería & Colima & $57 \pm 9$ & $105.8 \pm 0.3$ \\
CriollaTala & Jalisco & $64 \pm 2$ & $80.0 \pm 2$ \\
Tecomán & Colima & $42 \pm 4$ & $37.0 \pm 1$ \\
El Bordo & Colima & $34 \pm 3$ & $76.6 \pm 0.3$ \\
\hline
\end{tabular}

Fuente: (Borrás, 2015)

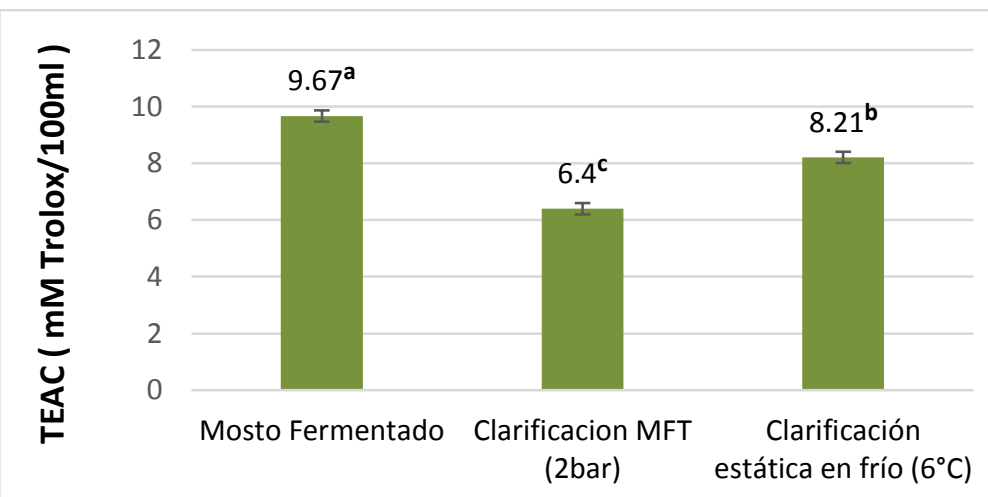

Figura 6. Actividad antioxidante expresada en el proceso de clarificación por MTF y por clarificación estática en frío

Las medias con letras distintas indican diferencia significativa de acuerdo con la prueba de Tukey ( $p$ $<0.05), \mathrm{n}=4 \pm$ desviación estándar

\subsection{Análisis físico químico del vino}

En la Tabla 6 se presentan los resultados obtenidos de los análisis físico-químicos del vino de flor de Jamaica clarificado con la mayor capacidad antioxidante y contenido de polifenoles. Los análisis fueron realizados en Seidlaboratory Cía.Ltda, laboratorio acreditado bajo normas ISO/IEC 17025, que determinaron que el vino clarificado estáticamente en frío cumple con la norma INEN NTE 374 para vino de frutas.

Tabla 6. Análisis físico-químicos del vino de flor de Jamaica

\begin{tabular}{cccc}
\hline \multicolumn{4}{c}{ Análisis fisico-químicos } \\
\hline Parámetros & Unidades & Resultado & Método de Referencia \\
\hline Grado alcohólico & $\circ \mathrm{GL}$ & 6 & INEN 340 \\
Metanol & $\mathrm{mg} / \mathrm{l}$ & 14.88 & INEN 347 \\
Anhídrido sulfuroso libre & $\mathrm{g} / \mathrm{l}$ & 0.09 & INEN 357 \\
Anhídrido sulfuroso total & $\mathrm{g} / \mathrm{l}$ & 0.13 & INEN 356 \\
\hline
\end{tabular}




\subsection{Medición de color}

En la Figura 7, se observan los promedios de las mediciones de absorbancia para espectrofotometría del proceso de vinificación de los cálices de flor de Jamaica (cálices, mosto sin fermentar, mosto fermentado), clarificación en frío a $6^{\circ} \mathrm{C}$ por seis días y del proceso de microfiltración tangencial (MFT) a 2 bar (retenido, permeado), en función del valor de las absorbancias medidas obtenidas de un barrido del espectro electromagnético visible (380 a $780 \mathrm{~nm}$ ); se comprobó que los procesos anteriormente mencionados poseen su mayor absorbancia dentro del espectro visible comprendido entre 500 y 540 (nm) aproximadamente con lo cual se establece que el color rojo predomina en las muestras y demostrado a través del método de Glories al comparar las longitudes de onda para 420 $\mathrm{nm}$ responsable del color amarillo, $520 \mathrm{~nm}$ del color rojo y $620 \mathrm{~nm}$ para el color azul (Figura 8 ), los cálices con un $62.84 \%$, el mosto sin fermentar un $71.49 \%$, el mosto fermentado un $65.86 \%$, mientras que el vino microfiltrado tiene $59.21 \%$ y el vino microfiltrado en frío en $63.86 \%$.

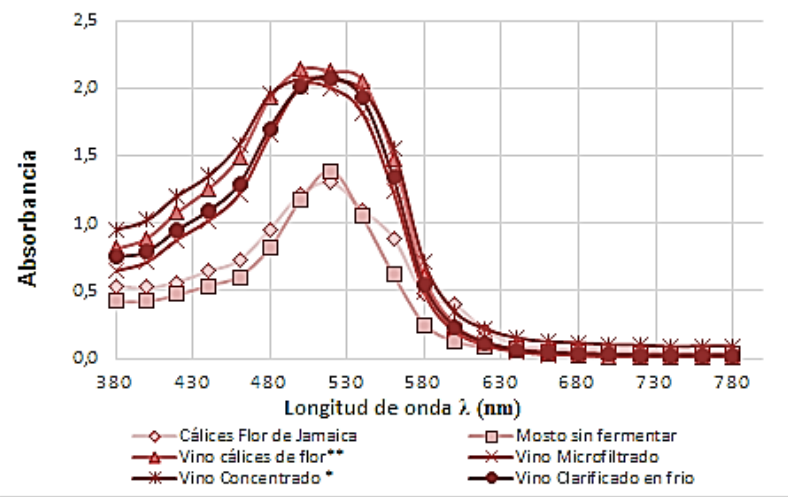

Figura 7.Comportamiento de las muestras estudiadas a diferentes absorbancias

${ }^{*}$ Representa el retenido

** Representa el mosto fermentado y la alimentación

El vino clarificado por MFT a 2 bar reporta un valor de 2.0 a una longitud de onda $\lambda$ de $520 \mathrm{~nm}$ mientras que el vino clarificado en frío de 2.084; valores relacionados a lo expuesto en el contenido de polifenoles totales. Garaguso \& Nardini (2015) menciona que las diferencias de color observadas en diferentes tipos de vinos se atribuyen a los cambios en los compuestos fenólicos extraídos de la materia prima durante la vinificación y maduración, además de tratamientos adicionales para aumentar su calidad.

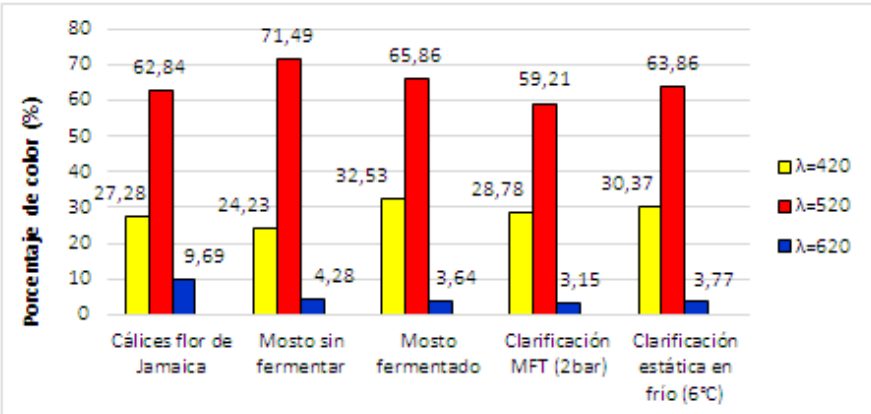

Figura 8. Porcentaje de color en el proceso de Vinificación y Clarificación por MFT y por frío

En relación con la intensidad de color (IC) se determinó que existen diferencias significativas $(p<0.05)$ entre las muestras (Figura 9), los cálices de flor de Jamaica presentan un IC de 2.072 valor próximo al obtenido por Babalola, Babalola \& Aworh (2001) de 2.102. El vino posee una intensidad de color de 3.324 superior a la reportada por Muñoz, 
Fernández, Ramos \& Alvarado (2007) de 0.541 en Tempranillo-Malbec-Cabernet Sauvignon, y 0.862 en Malbec-Merlot. Garaguso \& Nardini (2015) menciona que en vinos jóvenes y con períodos de almacenamientos cortos, la tonalidad varía en matices rojosvioláceos por la presencia de antocianinas, el vino clarificado en frío presenta una mayor intensidad de color en comparación al vino clarificado por microfiltración tangencial.

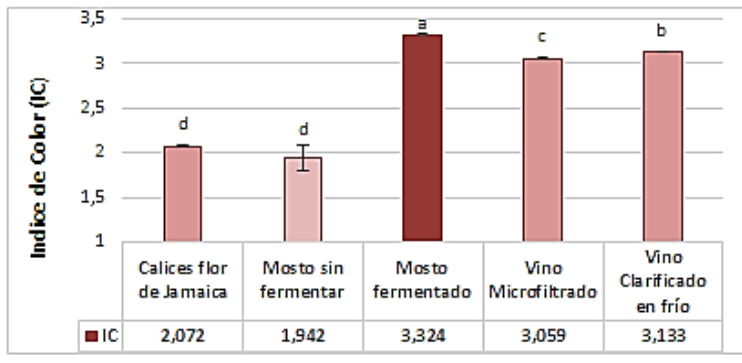

Figura 9. Análisis del Anova simple para intensidad de color (IC)

Las medias con letras distintas indican diferencia significativa de acuerdo con la prueba de Tukey $(p<0.05), n=2 \pm$ desviación estándar

El vino microfiltrado posee una intensidad de color de 3.059 inferior a 9.56 en Cabernet Sauvignon reportada por Arriagada, Saenz \& Bordeu (2005) quien establece que durante la microfiltración tangencial hay una reducción del $2.2 \%$ de la intensidad de color con respecto al mosto fermentado, describe que tal reducción se crea por la disminución del $4.8 \%$ de la concentración de taninos, fenómeno que se produce por la adsorción de los tanino en la membrana, el fenómeno de adsorción también lo reportó Ulbricht, Ansorge, Danielzika, König \& Schuster (2009).

El vino obtenido por clarificación en frío a $6{ }^{\circ} \mathrm{C}$ presentó una intensidad de color de 3.133 valor próximo al mosto fermentado, y similar a lo reportado por Cisse, Vaillant, SoroDoudjo, Reynes \& Dornier (2011), que concluyen que a temperaturas bajas no se presenta degradación exponencial de los pigmentos.

\subsection{Medición de turbidez}

Los resultados obtenidos sobre la turbidez determinaron diferencias significativas entre el proceso de vinificación de los cálices (mosto sin fermentar, mosto fermentado y cálices) y el proceso de clarificación por frío y por microfiltración a 2 bar (Tabla 7).

Tabla 7.Turbidez en el proceso de vinificación y clarificación por MFT y por frío

\begin{tabular}{lcc}
\hline \multicolumn{1}{c}{ Muestra } & $\begin{array}{c}\text { Parámetro } \\
\text { de trabajo }\end{array}$ & $\begin{array}{c}\text { Unidades nefelométricas } \\
\text { de turbidez (NTU) }\end{array}$ \\
\hline Mosto sin fermentar & $20^{\circ} \mathrm{C}$ & $252.5^{\mathrm{c}} \pm 2.12$ \\
Mosto Fermentado* & $20^{\circ} \mathrm{C}$ & $748.00^{\mathrm{b}} \pm 0.71$ \\
Vino Microfiltrado & $2 \mathrm{bar}$ & $1.31^{\mathrm{e}} \pm 0.01$ \\
Vino Concentrado** & $2 \mathrm{bar}$ & $>1000^{\mathrm{a}}$ \\
Vino Clarificado por frío & $6^{\circ} \mathrm{C}$ & $61.73^{\mathrm{d}} \pm 0.04$ \\
\hline${ }^{*}$ Representa la muestra de alimentación en el microfiltrado y de clarificación \\
estático por frío día 0 \\
${ }^{* *}$ Representa el vino retenido en el proceso de microfiltración \\
Las medias con letras distintas indican diferencia significativa de acuerdo con la \\
prueba de Tukey ( $\mathrm{p} \leq 0.05), \mathrm{n}=2 \pm$ desviación estándar
\end{tabular}

El proceso de microfiltración tangencial eliminó el $99.82 \%$ de la turbidez en el mosto fermentado a 2 bar, mientras que el proceso de clarificación estática en frío eliminó el $91.75 \%$ de la turbidez, un porcentaje mayor al $85 \%$ reportado por Ancín, Ayestarán \& Garrido (1996). La limpidez de un vino parámetro de calidad está relacionado con su turbidez, durante el proceso de microfiltración tangencial a 2 bar se obtuvo una turbidez de 1.3 NTU. Se determinó que el mosto fermentado es un vino brillante según lo 
establecido por El Rayess, Albasi, Bacchin, Taillandier \& Mietton (2011) que indican que independientemente de la turbiedad inicial del vino, la turbidez del permeado es siempre inferior a 1.5 NTU indicativo de un vino brillante.

\section{Conclusiones y recomendaciones}

Se determinó que durante el proceso de vinificación de los cálices frescos la fermentación es una etapa crítica en la liberación de compuestos fenólicos aumentando la contracción de polifenoles totales de $79.13 \mathrm{mg}$ ácido Gálico/100ml (mosto sin fermentar) a $144.15 \mathrm{mg}$ ácido Gálico/100ml (mosto fermentado), en el proceso de clarificación en frío a $6{ }^{\circ} \mathrm{C}$ por seis días, obtuvo un mayor contenido de polifenoles $139.32 \mathrm{mg}$ ácido Gálico/100ml, de actividad antioxidante de $8.21 \mathrm{mM}$ Trolox/100; se removió solo el $5 \%$ de los compuestos fenólicos en comparación con el proceso de clarificación por microfiltración tangencial a 2 bar que obtuvo $116.88 \mathrm{mg}$ ácido Gálico/100 ml, $6.40 \mathrm{Mm}$ Trolox/100 ml, al remover el $18 \%$ de compuestos fenólicos.

Se determinó que durante el proceso de microfiltración tangencial el flujo de permeado disminuye de acuerdo con la colmatación de la membrana, fenómeno que se desarrolla principalmente por los compuestos presentes en el vino, además de las condiciones de operación y tipo de membrana.

A partir de los resultados obtenidos de la capacidad antioxidante y contenido de polifenoles se evaluaron las características fisicoquímicas del vino y se determinó que el vino clarificado en frío cumple con la norma técnica para vino de frutas INEN NTE 374, al presentar $6^{\circ} \mathrm{GL}, 14.88 \mathrm{mg} / \mathrm{l}$ de metanol, $0.09 \mathrm{~g} / \mathrm{l}$ anhídrido sulfuroso libre y $0.13 \mathrm{~g} / \mathrm{l} \mathrm{de}$ anhídrido sulfuro total.

El análisis de color mediante espectrofotometría y por el método de Glories determinó que los dos procesos de clarificación tienen un color rojo oscuro, característico de los vinos tintos.

Se determinó que el vino obtenido por microfiltración tangencial a 2 bar presenta 1.31 NTU lo cual mejora su aspecto de calidad en comparación con el vino clarificado en frío que reportó 61.73 NTU.

\section{Bibliografía}

Ancín, C., Ayestarán, B., \& Garrido, J. (1996). Sedimentation clarification of Garnacha musts. Consumption of amino acids during fermentation and aging. Food research international, 29(13), 345-353. Recuperado el 23 de noviembre del 2016, de: https://www.researchgate.net/publication/

248425277 Sedimentation clarification of Garnacha musts Consumption of ami no_acids_during_fermentation_and_aging.Doi:10.1016/0963-9969(96)00034-8.

Arriagada, J., Saenz, C., \& Bordeu, E. (2005). Membrane filtration effects on aromatic and phenolic quality of Cabernet Sauvignon wines. Journal of Food Engineering, 363368. Recuperado el 26 de noviembre del 2016, de http://www.sciencedirect.com/science/article/ pii/S0260877404002936.Doi:http://dx.doi.org/10.1016/j.jfoodeng.2004.06.011

Babalola, S., Babalola, A., \& Aworh, O. (2001). Compositional attributes of the calyces of roselle (Hibiscus Sabdariffa L.). Journal of Food Technology in Africa, 133-134. Recuperado el 26 de octubre del 2016 de: http://www.ajol.info/index.php/jfta/article/view/19306

Bhave, R. R. (2014). Fermentation and Biochemical Engineering. En H. C. Vogel, \& C. M. Todaro. Fermentation and biochemical engineering handbook: principles, process design and equipment (págs. 149-180). New Jersey: William Andrew . Recuperado el 24 de noviembre del 2016, de: http://www.sciencedirect.com/science/article/pii/ 0260877484900372 . 
Bimpilas, A., Tsimogiannis, D., Balta, K., Lymperopoulou, T., \& Oreopoulou, V. (2015). Evolution of phenolic compounds and metal content of wine during alcoholic fermentation and storage. Food Chemistry, 178(1), 164-171.Recuperado el 20 de diciembre del 2016, de: http://www.sciencedirect.com/science/article/pii /S030881461500 104. Doi:http://dx.doi.org/10.1016/j.foodchem.2015.01.090

Borrás, I., Fernández, S., Arráez, D., Palmeros, P., Del Val-Díaz, R., Andrade, I., Segura, A. (Julio de 2015). Characterization of phenolic compounds, anthocyanidin, antioxidant and antimicrobial activity of 25 varieties of Mexican Roselle (Hibiscus sabdariffa). Industrial Crops and Products, 69, 385-394. Recuperado el 12 de noviembre del 2016, de:doi:http://dx.doi.org/10.1016/j.indcrop.2015.02.053

Cassano, A., Conidi, C., \& Drioli, E. (30 de Enero de 2010). Physico-chemical parameters of cactus pear (Opuntia ficus-indica) juice clarified by microfiltration and ultrafiltration processes. 250(3), 1101-1104. Recuperado el 7 de diciembre del 2016, de: http://www.sciencedirect.com/science/article/pii/S0011916409011497.Doi:10.1016/j. desal.2009.09.117

Castañeda, R., \& Cáceres, A. (2014). Compuestos bioactivos y propiedades terapéuticas de los cálices de rosa de Jamaica (Hibiscus sabdariffa Linn). Revista Científica de la Universidad de San Carlos de Guatemala. Laboratorio de Investigación de Productos Naturales (LIPRONAT), 7-24 .Vol. 24.Recuperado el 24 de octubre del 2016, de: http://revistaiiqb.usac.edu.gt/index.php/revista_cientifica/article/view/364.

Cisse, M., Vaillant, F., SoroDoudjo, Reynes, M., \& Dornier, M. (2011). Crossflow microfiltration for the cold stabilization of roselle (Hibiscus sabdariffa L.). Journal of Food Engineering, 20-27. Recuperado el 7 de diciembre del 2016 de: http://dx.doi.org/10.1016/j.jfoodeng.2011.04.001

El Rayess, Y., Albasi, C., Bacchin, P., Taillandier, P., \& Mietton, M. (2011). Cross-flow microfiltration of wine: Effect of colloids on critical fouling conditions. Journal of Membrane Science, 177-186. Recuperado el 7 de diciembre del 2016 de: http://www.sciencedirect.com/science/article/pii/S0376738811007253.Doi: http://dx.doi.org/10.1016/j.memsci.2011.09.037

Ennouri, M., Ben, I., Ben, H., Lafforgue, C., Schmitz, P., \& Ayad, A. (2015). Clarification of purple carrot juice: analysis of the fouling mechanisms and evaluation of the juice quality. Journal of Food Science and Technology, 52(5), 2806-2814. Recuperado el 8 de diciembre del 2016 de: https://www.ncbi.nlm.nih.gov/pubmed/25892778. Doi:10.1007/s13197-014-1323-9.

Fernández, S., Rodríguez, I. C., Beltrán, R., Pasini, F., Joven, J., \& Fernández, A. (2011). Quantification of the polyphenolic fraction and in vitro antioxidant and in vivo antihyperlipemic activities of Hibiscus sabdariffa aqueous extract. Food Research International, 44(5), 1490-1495. Recuperado el 15 de noviembre del 2016 de: http://www.sciencedirect.com/science/article/pii/S0963996911001980.

Garaguso, I., \& Nardini, M. (2015). Polyphenols content, phenolics profile and antioxidant activity of organic red wines produced without sulfur dioxide/sulfites addition in comparison to conventional red wines. Food Chemistry, 336-342. Recuperado el 28 de noviembre del 2016 de: http://www.sciencedirect.com/science/article/pii /S0308814615001806

Ifie, I., Marshall, L. J., Ho, P., \& Williamson, G. (2016). Hibiscus sabdariffa (Roselle) Extracts and Wine: Phytochemical Profile, Physicochemical Properties, and Carbohydrase Inhibition. Journal of Agricultural and Food Chemistry, 64(24), 4921-4931. Recuperado el 19 de noviembre del 2016 de https://www.ncbi.nlm.nih.gov/pubmed /2722 6105. Doi:10.1021/acs.jafc.6b01246.

Jackson, R. S., Kilcast, D., \& Subramaniam, P. (2011). Shelf life of wine. Food and beverage stability and shelf life, 540-570. Recuperado el 22 de noviembre de 2016, de https://www.researchgate.net/publication/282934821_Shelf_life_of_wine.

Medina, R., \& Sumaya, M. T. (2013). Actividad antioxidante de extractos de cálices deshidratados de 64 variedades de jamaica (hibiscus sabdariffa l.) en función de 
fenólicos y antocianinas totales. Revista Ciencias Técnicas Agropecuarias, Vol. 22, No. Esp., (diciembre, pp. 41-44).Recuperado el 23 de noviembre del 2016 de http://scielo.sld.cu/scielo.php?script=sci_arttext\&pid=S2071-00542013000500006

Re, R., Pellegrini, N., Proteggiente, A., Pannala, A., Yang, M., \& Rice-Evans, C. (1999). Antioxidant activity applying an improved abts radical cation decolorization assay. Free Radical Biology \& Medicine, 26(9/10), 1231-1237.Recuperado el 13 de noviembre del 2016 de: http://dx.doi.org/10.1016/S0891-5849(98)00315-3

Sáyago, S., \& Goñi, I. (2010). Hibiscus sabdariffa L: Fuente de fibra antioxidante. Archivos latinoamericanos de nutrición, 79-84. Vol. 60 № 1.Recuperado el 29 de octubre del 2016 de http://www.scielo.org.ve/scielo.php?script=sci_arttext\&pid=S000406222010000100012. 